\title{
Innovative Design Thinking Process with TRIZ
}

\author{
Kyeongwon, LEE \\ Dept. of Mechanical Design Engineering, Korea Polytechnic University, Korea \\ Siheung City, Gyeonggi-Do, 429-793, Korea \\ $l \mathrm{kw} @ \mathrm{kpu} \cdot \mathrm{ac} \cdot \mathrm{kr}$
}

\begin{abstract}
This paper describes an innovative design thinking process with simplified TRIZ that can be used to resolve contradictions in all domains with words such as "dilemma", "conflict", "contradiction" and "paradox". The design thinking process that have been used at d.school at Stanford and Potsdam University are popular as a human-centered innovation process with "Empathy" and "Define" stages for human-centered problem finding. However, many results may be not innovative because it uses mostly "Group brainstorming" at "Ideate" stage.

TRIZ users have complained that the Russian conventional TRIZ is so difficult to learn and apply. They consider that it is useful in manufacturing and mechanical fields mostly. This paper suggests an innovative design thinking process with simplified and generally usable the stepby-step TRIZ. Its effectiveness of the innovative design thinking process with the simplified and step-by-step general TRIZ is explained in the development case study, a smart wind free air conditioner.
\end{abstract}

Keywords: Innovative Design Thinking, Simplified TRIZ, Step-by-step TRIZ

\section{Introduction}

Among several conceptual design methodologies, Design thinking and TRIZ are popular in the world. Design thinking is a process of a human-centered innovation with collaboration of team members. TRIZ is technologically oriented methodology to resolve some contradictions using knowledge databases.

When it comes to TRIZ, newcomers are confronted with highly sophisticated tools and a very time-consuming learning process as explained in Claudia Hentschel and Alexander Czinki' paper [1]. They presented that Design Thinking as a door-opener for TRIZ is efficient for newcomers to understand and use TRIZ as a systematic innovation method ology.

There are several related papers by searching both "design thinking process and TRIZ" in scholar.google.com [2,3,4]. TRIZ is Russian acronym of "Theory of Inventive Problem Solving". There are so many problem-solving tools of TRIZ than tools for human-centered problem finding. It is very important to find and define the human-centered problem and project in highly competitive economics these days because of faster changes of customer's needs. TRIZ that has used mostly in mechanical and manufacturing fields gives just some conceptual ideas and sometimes looks like innovative, but still vague ideas so that TRIZ users do not confirm that the ideas are working well or not in short time.

The Design thinking process can compensate the weakness of TRIZ well. The 
"Empathize" and "Define" step of the Design thinking process are effective steps for compensating TRIZ for finding the human-centered problem as a "Pre-TRIZ".

The fast and cheap "Prototype" step of the Design Thinking process is serial step for communicating the TRIZ ideas much better with others and then, showing effective implementation of conceptual ideas by TRIZ as a "Post-TRIZ".

Vice versa, the Design thinking process has big shortage on generating innovative ideas in the "Ideate" step because it depends on group brainstorming method by all participants. Therefore, TRIZ and Design thinking process can compensate each other.

Design thinking process has strong points in problem finding with collaborative team play. Simplified TRIZ with interesting education samples are suitable to improve the creativity of even students at middle and high school better.

This paper describes a general-use innovative design thinking process with simplified and step-by-step TRIZ that can be used to resolve contradictions in all domains with words such as "dilemma", "conflict", "contradiction".

Its effectiveness is explained in a development case study, the smart wind free air conditioner at SAMSUNG Electronics.

\section{Design Thinking Process}

The design thinking process first defines human-centered problem carefully and then, implements the solutions, always with the needs of the user demographic at the core of concept development [5]. This process focuses on needs finding, understanding, creating, thinking, and doing. At the core of this process is a bias towards action and creation: by creating and testing something, you can continue to learn and improve upon your initial ideas. The design thinking process at d.school of Stanford University is most popular in the world. It consists of these 5 steps as shown in Fig. $1 .:$

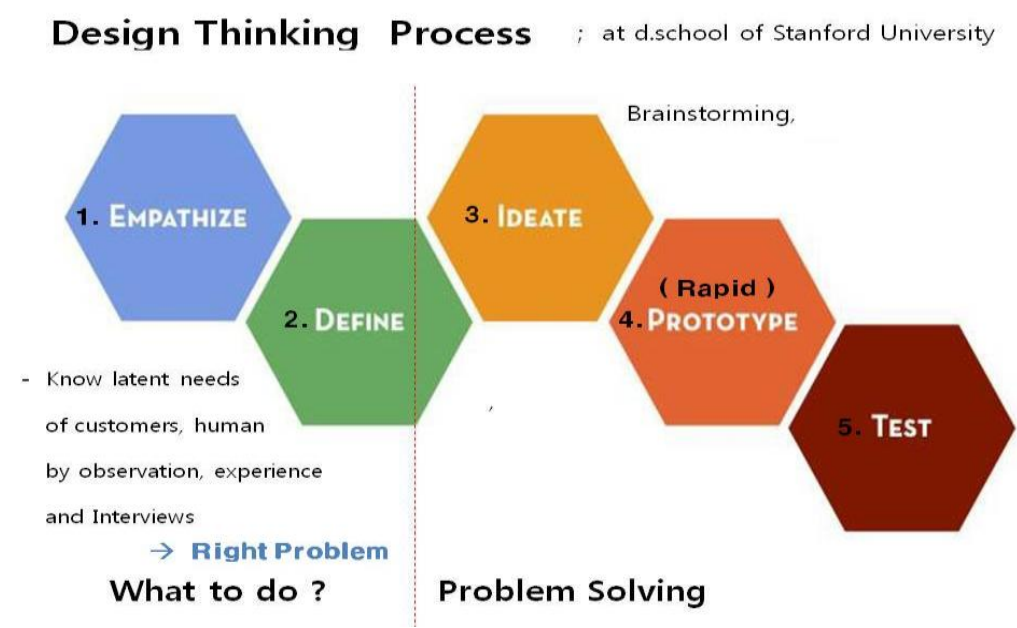

Fig. 1. 5 steps of design thinking process at d.school, Stanford University [5] 
1) "EMPATHIZE" step: Work to fully understand the experience of the user for whom you are designing. Do this through observation, interaction, and immersing yourself in their experiences

2) 'DEFINE' step: Process and synthesize the findings from your empathy work to form a user point of view that you will address with your design.

3) "IDEATE" step: Explore a wide variety of possible solutions through generating a large quantity of diverse possible solutions, allowing you to step beyond the obvious and explore a range of ideas. In many cases, the methods to generate ideas almost depend on brainstorming of multidisciplinary team.

4) "PROTOTYPE" step: Transform your ideas into a physical form rapid and cheaply so that you can experience and interact with them and, in the process, learn and develop more empathy.

5) "TEST" step: Try out high-resolution products and use observations and feedback to refine prototypes, learn more about the user, and refine your original point of view.

The design thinking refers to the way designers solve problems, develop unique thought streams, visualize ideas quickly and then, start to modify the draft plans again after reviewing them as shown in Fig. 2.

It has been more popular in the world including Korea than TRIZ after big companies such as SAP, Google, Airbnb, IBM and Siemens have started to utilize it as collaborative working ways.

Design Thinking ; Collaboration (Team) + Communication (Visual) + Human-Centered Design

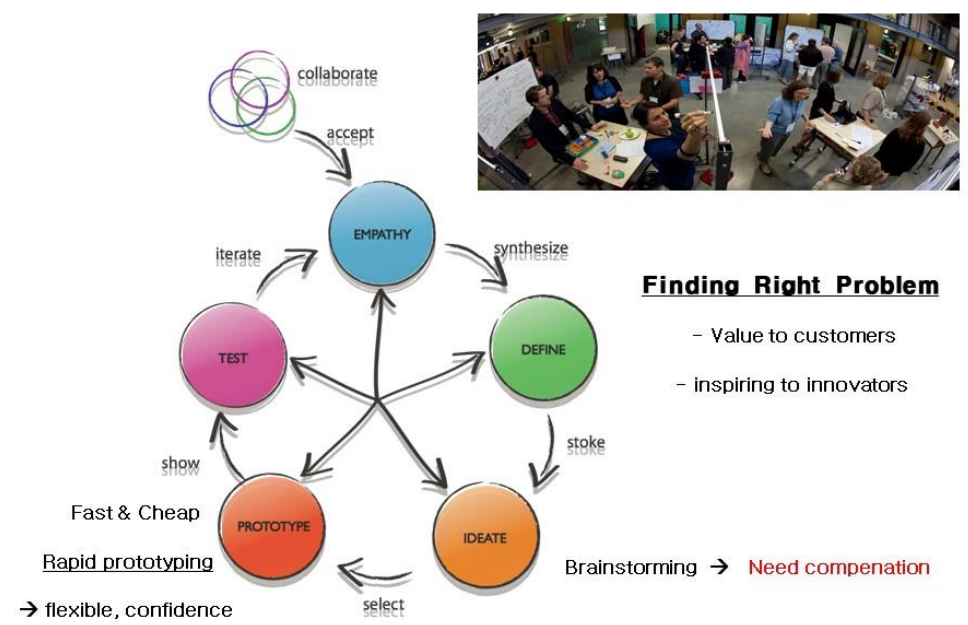

( Design thinking Process of d.school, Stanford University )

Fig. 2 Design thinking process of d.school, Stanford University [5] 
It defines problems through the "Empathy" for real users, produces many possible ideas for problem solving through group brainstorming, and then produces an optimal number of ideas among them. It uses the several methods for empathy to identify potential user's latent needs using careful observation and deep interviews etc. with real users to identify problems on the viewpoint of users.

It forms a team of people from different fields to collect diverse ideas to solve problem with diverse viewpoints, knowledge and experiences.

For much better communication, it uses visual communication methods such as sharing the simple drawings on Post-its attaching to big panels and the fast prototype with low resolution with cheap materials, so that all participants can exchange diverse opinions, communicate freely and understand the ideas better and quickly.

However, the idea-generating phase (called by "IDEATE" step) is mainly based on the use of light idea-generating methods, such as group brainstorming and sometimes MINDMAP and SCAMPER as well. The quality and innovativeness of ideas are still poor in many applications.

Design thinking users have complained that design thinking looks like one of facilitating methods with funny ice braking and recreations without innovative results in Korea. Because design thinking experts have introduced and explained just mostly good innovation results and case studies from Stanford University and IDEO company. The design thinking or HCI (Human-computer interaction) promotion teams inside big companies in Korea such as SK groups have been disappeared by poor innovative business results for several years.

\section{$3 \quad$ Innovative Design Thinking Process with Simplified TRIZ}

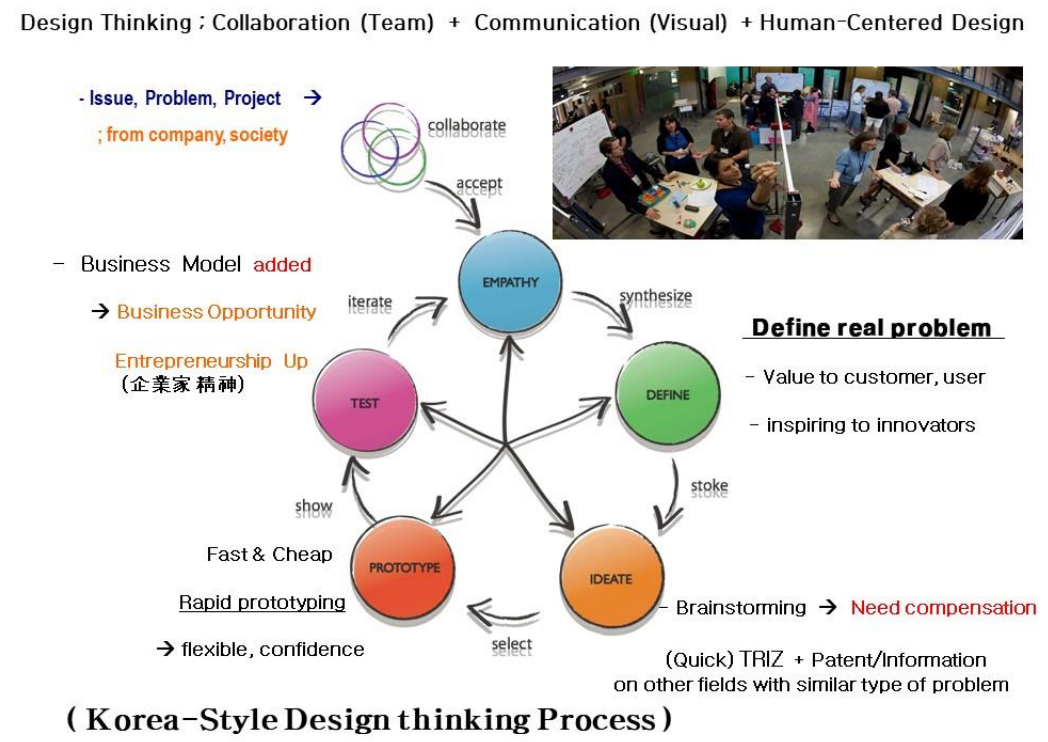

Fig. 3 Innovative design thinking process, "Korea-style design thinks process" 
TRIZ has no steps to include human-oriented latent and changing needs. The human-centered "Empathy" step of Design thinking process uses careful observation of user's vivid actions, user's intensive interview and solver's experience. The step knows and defines problem solvers the human-centered problem correctly that user and customer have latently. It is much effective steps for compensating TRIZ for finding human-centered problem well as "Pre-TRIZ" stage. For nice and more implementations from ideas by TRIZ to practical innovation and commercialized products or service in market, the human-centered problem definition for customers can give the innovator and problem solver the inspiring to challenge. In addition, TRIZ is one method and process for just conceptual design stage. It gives just conceptual ideas than more concrete ideas. Hence TRIZ users may not conformed whether the conceptual ideas by TRIZ can be implemented by technical and business aspects in the field well or not. They need some additional steps to explain the TRIZ ideas better to others. For instance, some TRIZ promotion team at big companies such as SAMSUNG in Korea have matched and linked both idea generators by using TRIZ and different domain experts with computer aided simulation and analysis, making mock-up, simple experiment. The fast and cheap "Prototype" step of the design thinking process between idea generation stage of TRIZ and more expensive working prototype can compensate TRIZ as "Post-TRIZ" stage. For more implementation better and time-reduction for $\mathrm{R} \& \mathrm{D}$, the fast, cheap and low-level prototyping of Design thinking process is very important for TRIZ users who may not give strong confidence on their conceptual ideas. At early stage they can know technically feasibility before high-level and expensive prototyping product or service. Complementing innovative ideas to the "IDEATE" stage of conventional design thinking by simplified and step-by-step TRIZ first and then, conventional complex TRIZ knowledges more as shown in Fig. 3.

TRIZ is suitable method to resolve any kinds of contradictions, so-called dilemma in other fields besides Engineering problems. By the way, TRIZ beginners and general persons have some difficulty to model the right contradiction systematically. In addition, they have complained that the conventional TRIZ has so many tools such as 40 inventive principles, 76 standard and complex ARIZ process.

The simplified TRIZ, so-called the "Quick TRIZ" process with step-by-step conflict diagram in T.O.C (Theory of Constraints) was devised as follows [6]. As shown in the Fig. 4, it asks to describe the cause to remove the main cause of problem into box B and then, write down one first good idea (as the remedy) by users themselves or sometimes by group brainstorming in design thinking process, which can remove the cause in box B, into box D.

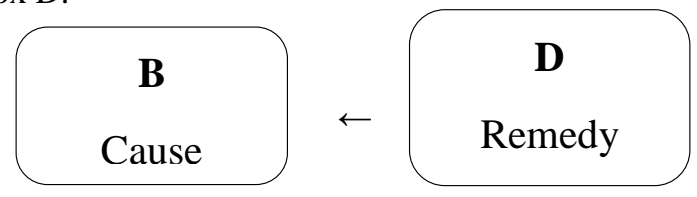

Fig. 4. Pair with one main cause and its remedy idea to remove the cause 
Then, it asks a problem solver to describe the new problem generated by implementing the first idea (in many cases, the first idea generates the new other problem). In design thinking process, good ideas may generate a new other problem such as an expensive idea, heavier problem and other bad side-effect to other parts.

The box - $\mathrm{C}$ will be filled up the contents on the other new problem generated, that is to remove the new problem as shown in Fig. 5.

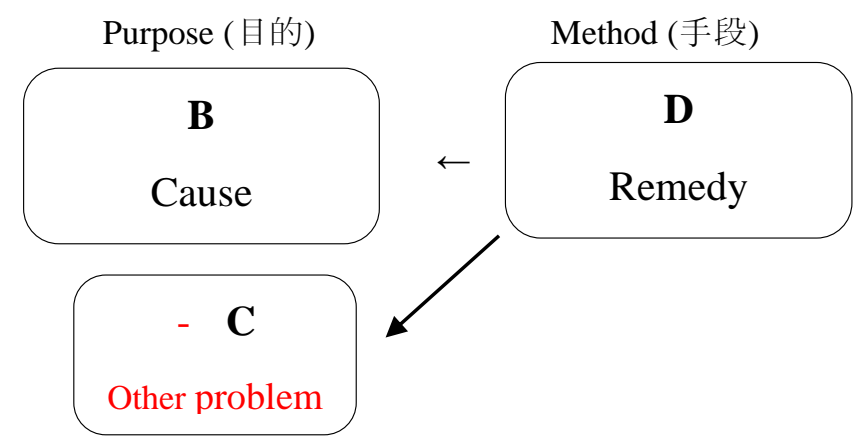

Fig. 5 New problem generated by the remedy idea to remove the cause

Through the progress, the first visual description on the contradictive problem is represented. In box D' shown in Fig. 6, the reverse (-) condition corresponds to the method to remove the cause should be described intentionally.

In other words, the minus or the reverse physical condition against the method in box D should be described in box D' as the physical contradiction of TRIZ.

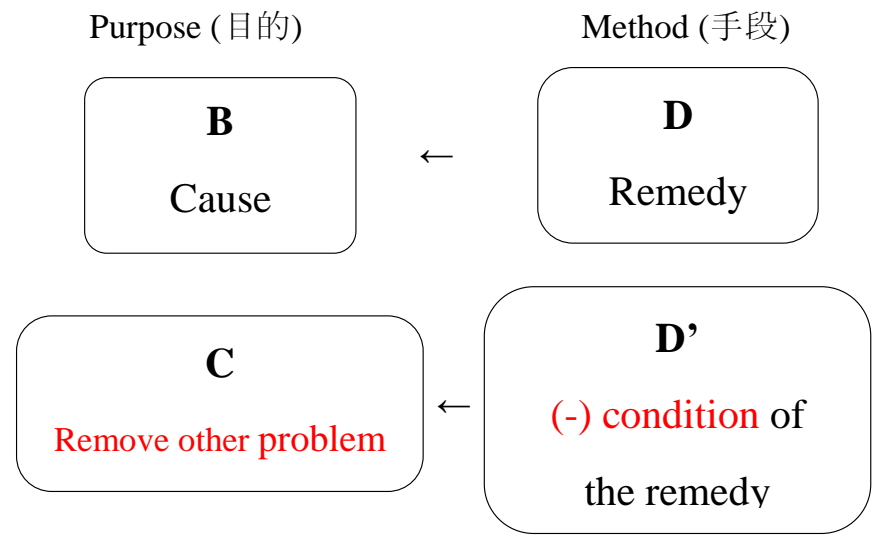

Fig. 6 Reverse condition of the remedy to remove new other problem

As the last step the box A will be filled with the Ideal Final Result (IFR) of TRIZ, that satisfies the two contradictive purposes in box B and C "at no cost concept" shown in Fig.7. 


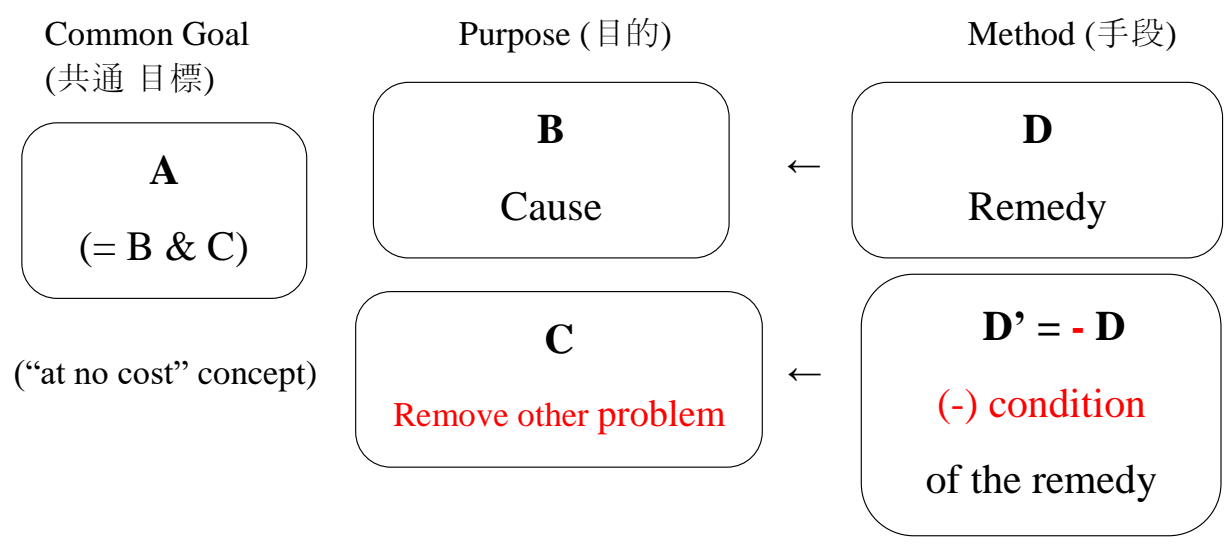

Fig. 7. Problem description in the Conflict Diagram in T.O.C.

TRIZ solvers can see the technical contradiction in box B and box C and the physical contradiction in box D and box D' corresponding the problem with the main cause, the method to remove the cause and the new problem generated by the method and the self-contradictory relation at the same time.

The author has realized through his over ten-year-experience in the field of TRIZ education and business that the terminology of the "contradiction" is what the TRIZ beginners find difficult. And it seems to be unfamiliar and hard to get the correct meanings of the physical contradiction or the technical contradictions and the differences between them as well.

That is why some Korean TRIZ experts call the contradiction the conflict for people working for non-technical field. In other words, they use the term, 'conflict' between two purposes (目的) instead of the technical contradiction in TRIZ and use the term, 'conflict' between two methods (手段) instead of the physical contradiction in TRIZ to make people more understandable.

And then, apply 3 separation principles and "Using other cheaper resources" simply as follows;

1) Generate ideas by applying "separation in time"

2) Generate ideas by applying "separation in space" including "separation in whole and parts"

3) Generate ideas by applying "separation on conditions" with difference of level, standard and condition with "if" and "otherwise"

4) Generate ideas by using other cheaper resources instead of 40 principles and standard solutions to escape from fixed and existing idea, means and method.

Notice that solvers intentionally describe the revere idea, means and method of D (-D) into the box D' to satisfy the contradictive purpose by removing the new other problem as shown in Fig. 7.

As common goal they add the contradictory purposes B and C into the box A. Thus, they can model the right physical contradiction step-by-step from the idea by 
group brainstorming of the "IDEATE" step of the design thinking process that has been applied to all human related domains with mechanical and manufacturing fields.

If you do not satisfy the ideas by the simplified and step-by-step TRIZ above, use conventional Russian TRIZ knowledges serially more and more.

The innovative design thinking process with TRIZ can include the lean business model canvas [7] with the information/ patent search and user's journey map for real implementation commercially more as shown in Fig. 3. It is called by "Korea-style design thinking process" in Korea like "K-Pop (Korea Pop)" popular in the world.

\section{Case Study: Development of smart wind free air conditioner}

Since the first commercial use of air conditioner by the company, CARRIER in the early 20th century, LG Electronics has led the global market these days. Many patents and core technologies of air conditioners have already been existed in the United States, Europe, Japan, and LG Electronics.

To enter this market and then, compete with the leading companies as a follower company, it is necessary to have consistent efforts to recognize and reflect the needs and demands of consumers on the product more in advance. Many users of air conditioner or purchasing customers surveyed are likely to require general complaints such as a lot of power consumption, loudness and expensive price. Are there any other potential complaints and demands?

Design thinking recommends to interview deeply the "Extreme users " in the "EMPATH" phase to gain new perspectives on users. The extreme user is a small number of people who live, think, and consume differently than most users. SAMSUNG Electronics can get unstructured perspectives and insights from them, skin-sensitive extreme users for cold strong wind of air conditioner.

The product developed by capturing different perspectives on air conditioners is the smart wind free air conditioner [8].

The development team members of SAMSUNG had observed carefully more than 600 large hotels, shopping centers, air conditioning system of an airplane and interviewed deeply the mechanics who install and fix the air conditioner up as well as users of home-use air conditioner. They had identified some latent needs of users and then, considered the new product considering the related technologies and patents.

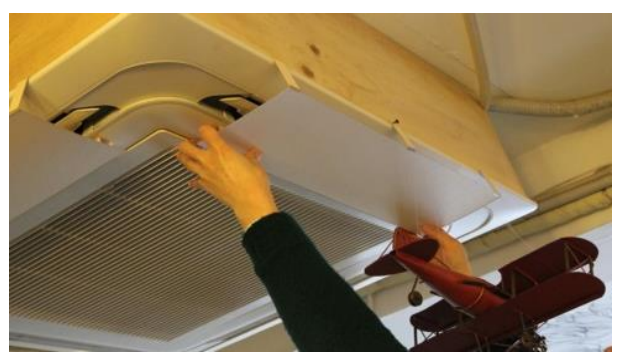

Fig. 8 Wind guide plate to divert cold wind from air conditioner to other direction 
They identified new target customers as extreme users who are very sensitive of cold strong wind to skins. Most ordinary users of air conditioner with the extreme users may remember the bad feelings from the cold strong wind of air conditioners to their skins.

Some users divert the direction of the wind of air conditioner from one side to the other by attaching extra wind-guiding wings not contacting it to their skin directly as shown as in Fig. 8.

However, it is likely to consume much more power due to poor cooling capabilities. The development team defined the problem with "How might we release the bad feeling of users who are very sensitive to cold strong wind from air conditioners while cooling the room down well simultaneously? "

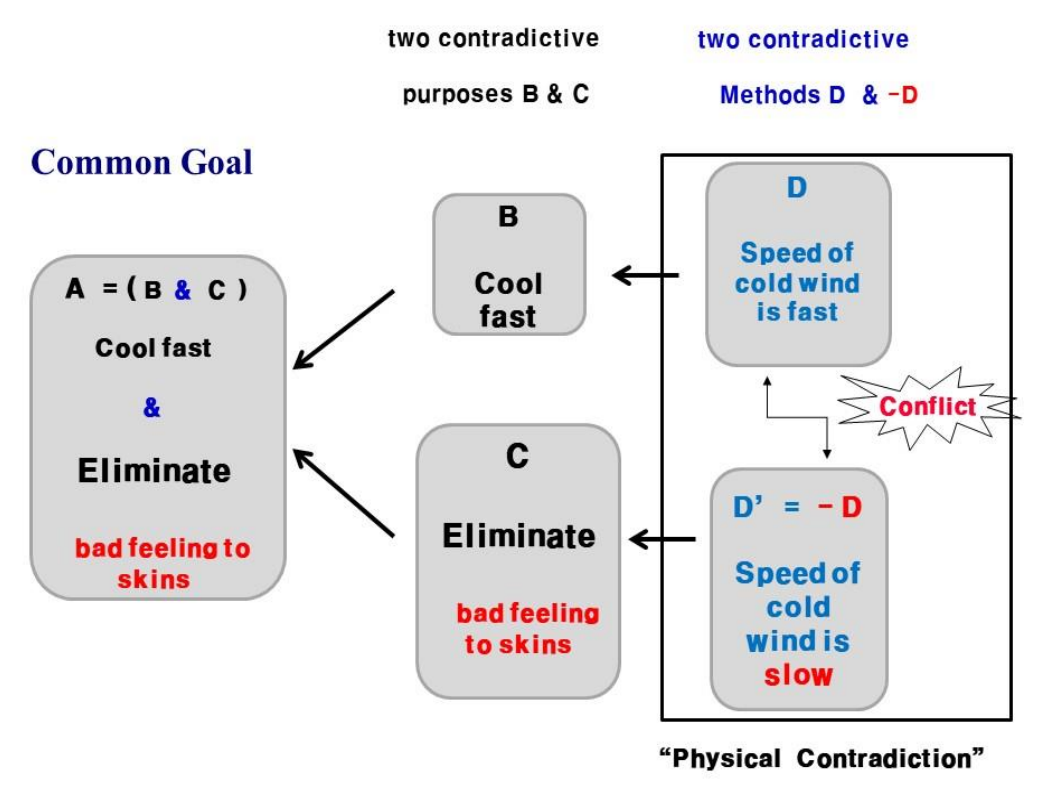

Fig. 9 Conflict diagram of physical contradiction with fast and slow cold winds

They came up with an idea and then, have made a prototype for complete product based on the design thinking process. They had big difficulty that there is no wind preventing touching the skin by the cold strong wind from air conditioner while if there is no wind, the air conditioner does not cool the room down any more.

It is a situation of contradiction and dilemma that air conditioners must blow the "fast" and "not fast (=slow)" cold wind simultaneously. The ideas should satisfy both two opposing purposes of keeping cooling down and eliminating the bad feeling to skin by the strong cold wind resolving the physical contradiction of TRIZ with "fast cold wind" and "slow cold wind" as shown in Fig. 9.

It is not easy to resolve these conflicts and contradictions by brainstorming alone. It is much better to apply the separation principles of TRIZ for resolving the physical 
conditions. The consumer wants to cool down fast when he turns on the air conditioner. After several minutes using the air conditioner, he wants to turn it off for preventing the bad feeling by blowing the cold wind to his skins. It is possible to come up with the idea by the "separation in time" principle of TRIZ. For example, there are fast cold wind of the air conditioner for first 10 minutes. After the 10 minutes there is not fast (=too slow) cold wind.

Where in nature are places to satisfy these contradictory demands for cooling are accomplished? The answer is the cold "cave" in summer.

Human skin does not feel the badness against wind with the low velocity air conditioners. It feels like there's no wind. According to the scientific survey the wind speed from air conditioners is about 2 meters per second. By careful measurement there is a weak wind in the cave with a wind speed of just $15 \mathrm{~cm}$ per second. It is not only the lack of fast wind in the cave, but also the breeze in the air conditioner's onefifteenth wind that makes it cool. When you combine it with the idea of the separation in time principle, it will satisfy users who want to feel cool like a traditional air conditioner and then, cool down like a cave after the 10 minutes. With this idea it develops detailed design and implementation with technical experts on air condition.

Since lowering the wind speed due to calm mode and extreme winds reduces the ability to cool the air conditioners down, it is important to ensure that the air conditioning capacity is maintained while lowering the wind speed significantly. Technically the cooling capacity is the multiplication of the discharge area with wind and wind speed.

In other words, if you want to achieve the same cooling capacity due to low wind speed you must make the air discharge area where air conditioners are produced very large.

\section{Separation in Time - After strong \& cold, weak wind}

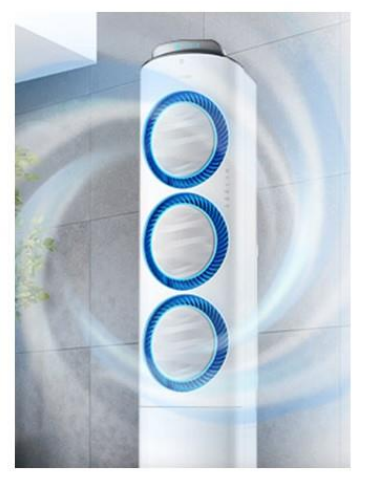

10 min. since turn-on

- Strong cold wind

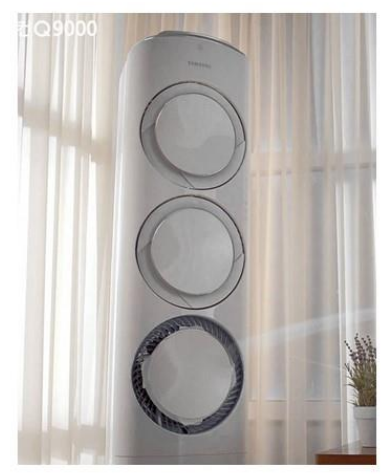

after 50 min. since turn-on

- Sweet \& weak wind

( as from whole body of audio speaker around )

Fig. 10 Air conditioner applied by "separation in time" principle 
To compensate for the wind speed that has decreased by $1 / 15$, how can we expand the discharge area of air conditioners by 15 times? Whereas conventional air conditioners produce wind from a limited area of air vents, the idea is improved as if the entire body of air conditioners were made from air conditioner blast device like audio speakers as shown in Fig. 10. Engineering knowledges on air conditioner resolve the contradiction of low wind speed and cooling capacity with the concept of TRIZ.

Eventually about 100,000 very small holes in the entire body of the air conditioner were created, creating a new type of air conditioner that creates a sense of calm to humans. Samsung's home-use air conditioner was released to Korea domestic market in 2016. Despite of expensive price, it has become a hit product selling more than 200,000 units first year in Korea. It has been exported massively in the world market as smart innovative air conditioner recently.

It introduced a new product and a new technology that can mark a new historic product and technology on over 100 years in the history of air conditioners by using the innovative design thinking process with TRIZ serially.

\section{Acknowledgement}

This work was partially supported by the Korea Foundation for the Advancement of Science and Creativity (KOFAC) and Korea Institute of Design Promotion (KIDP) grant funded by the Korea government, respectively.

\section{Conclusions}

The design thinking process of d.school of Stanford University has strong point for collective intelligence through interesting and funny actions by team members, visual communication between all participants and human's (user's) latent needs found by careful observation and deep interview real users at the "Empathy" stage.

But many users to apply it have complained that the idea is not innovative as the ideas are based on the participants ' group brainstorming. Even with innovative ideas, these innovative ideas become more expensive or heavier or have new other problems that make them less practical to apply. This new other problem situation will be a contradiction and a conflict. So TRIZ with the separation and invention principles can be used to resolve the contradictions, conflicts and dilemmas in any other domains.

By the way the conventional Russian style TRIZ is still quite difficult for ordinary people and beginners to learn and apply. Because there are so many complex problem tools of TRIZ. They are composed of many 40 invention principles that are suited to mostly mechanical field and manufacturing, 76 Standard Solutions with Substance Field Modeling and complex ARIZ process starting from defining the technical system. Especially it is difficult to define the conflicts and contradictions that are the real cause of the problem easily and step-by-step within several hours' TRIZ educations.

According to this innovative design thinking process with step-by-step TRIZ, ordinary people and beginners can learn only a few hours, but also model the conflicts systematically by using the conflict diagram of T.O.C. with familiar words. 
This simplified and step-by-step TRIZ may be applied to any general domains with contradictions, conflicts and dilemmas.

Even young students can easily memorize only four principles to resolve contractions with "separation in time, in space, on condition and using other cheaper resources in any other contradictive. Thus, this innovative design thinking process with TRIZ, specially simplified and step-by-step TRIZ is good harmonized conceptual design methodology compensating the strong and weak points of Design thinking and TRIZ each other, respectively.

The results after applying conceptual design methods such as design thinking and TRIZ are often just conceptual ideas. If we add them by the prototyping and business modelling canvas for showing the business opportunities more and starting a little business with the lean start-up concept [7], you'll see far more practical business results from the just conceptual ideas.

In the innovative design thinking process with TRIZ, we will add how to choose the attractive, trendy, social and business issues to find good problems better and build innovation culture of organizations and heighten the enthusiasm and willingness of the participants to innovate more.

\section{References}

1. Claudia Hentschel, Alexander Czinki: Design Thinking as a door-opener for TRIZ- Paving the way towards systematic innovation, TRIZ Future Conference 2013, Paris, France (2013)

2. Katja Thoring, Roland M. Müller: Understanding the Creative Mechanisms of Design Thinking: An Evolutionary Approach, Proceedings of the 2nd Conference on Creativity and Innovation in Design, pp. 137-147, Eindhoven, Netherlands, October 19-21 (2011)

3. Vinay Dabholkar: Design thinking vs TRIZ: A panel discussion, on July, 5 as a part of Next Gear Workshop, Catalign Innovation Consulting (www.catalign.com) (2012)

4. Colin M. Gray etc.: What is the Content of "Design Thinking"? Design Heuristics as Conceptual Repertoire, International Journal of Engineering Education, Vol. 32 (2016)

5. d.school of Stanford University Homepage, http://dschool.stanford.edu, last accessed 2018/04/14.

6. Kyeongwon LEE: Simple TRIZ Process "Quick TRIZ 2014" for non-technical fields with resolving the dilemma in business, service, government policy and social conflicts systematically, Proceedings of $14^{\text {th }}$ ETRIA World Conference TRIZ Future 2014, Lausanne, Oct. 29-31 (2014) and Journal of the European TRIZ Association, INNOVATOR, ISSN 1866-4180, 01/2014 Volume 01, pp.110-112 (2014)

7. Eric RIES: Lean startup, The lean startup : how today's entrepreneurs use continuous innovation to create radically success, $1^{\text {st }}$ edition, Currency (2011)

8. Ji-Young, KIM: Thinking wind free air conditioner with Design Thinking, KOSCA (KOrea Society of Creativity Application, www.kosca.net) Domestic Fall Conference 2016, Seoul (2016) 